\title{
Technè
}

La science au service de l'histoire de l'art et de la préservation des biens culturels

$44 \mid 2016$

Archives de l'humanité : les restes humains patrimonialisés

\section{Regard sur les restaurations anciennes et dérestauration : ce que les pratiques passées nous révèlent}

Examining old restoration and de-restoration issues: what we learn from past practices

\section{Laure Cadot}

\section{OpenEdition Journals}

Édition électronique

URL : http://journals.openedition.org/techne/1144

DOI : $10.4000 /$ techne. 1144

ISSN : 2534-5168

Éditeur

C2RMF

Édition imprimée

Date de publication : 1 novembre 2016

Pagination : $90-95$

ISBN : 978-2-7118-6339-6

ISSN : $1254-7867$

Référence électronique

Laure Cadot, «Regard sur les restaurations anciennes et dérestauration : ce que les pratiques passées nous révèlent », Technè [En ligne], 44 | 2016, mis en ligne le 19 décembre 2019, consulté le 26 juillet 2020. URL : http://journals.openedition.org/techne/1144 ; DOI : https://doi.org/10.4000/techne. 1144

\section{(c) $)(1)(9)$}

La revue Technè. La science au service de l'histoire de l'art et de la préservation des biens culturels est mise à disposition selon les termes de la Licence Creative Commons Attribution - Pas d'Utilisation Commerciale - Pas de Modification 4.0 International. 


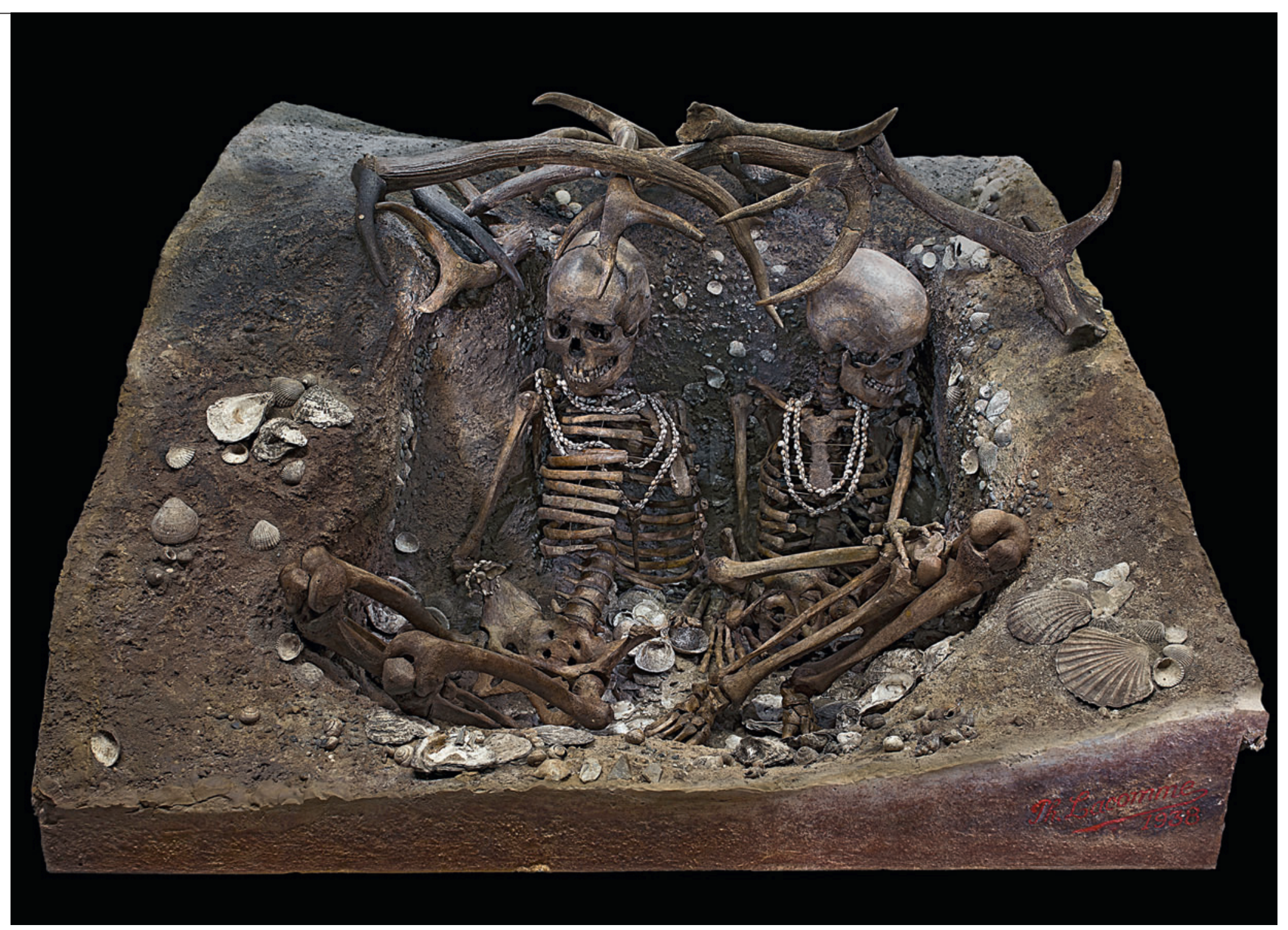

Fig. 1. La muséographie patrimonialisée de la sépulture de Téviec. Muséum d'histoire naturelle de Toulouse. @ D D. Descouens. 
Laure Cadot

\section{Regard sur les restaurations anciennes et dérestauration : ce que les pratiques passées nous révèlent}

Examining old restoration and de-restoration issues: what we learn from past practices

Résumé. Les préoccupations conservatoires concernant les restes humains dans les collections accompagnent depuis quelques dizaines d'années leur retour sur le devant de la scène scientifique et muséale. Du point de vue de la conservation-restauration, cette reconsidération patrimoniale incite à questionner, à l'appui de nos connaissances et de notre sensibilité contemporaine, les pratiques passées pour en comprendre les motivations et les enjeux dans le contexte de leur époque. Cet article propose, à la lumière de quelques exemples, d'esquisser non pas une histoire de la restauration des restes humains dans les institutions culturelles - qui reste à écrire -, mais un panorama de pratiques visant à orienter l'approche actuelle de ce domaine en pleine construction.

Mots-clés. Réparation, remontage, contamination, dérestauration, éthique, documentation.
Abstract. For decades, conservational preoccupations concerning human remains in collections have gone hand in hand with their return to the centre of the scientific and museum stage. From the conservation-restoration point of view, this reconsideration of their heritage status prompts questions - based on contemporary sensibility and knowledge - about past practices, in order to understand the reasons and challenges in the context of their era. In light of a few examples, this article proposes to outline, not a history of the restoration of human remains in cultural establishments (which remains to be written), but a panorama of practices which aims to guide the present approach to this emergent field.

Keywords. Repairs, remounting, contamination, de-restoration, ethics, documentation.

\section{Retour en humanité}

Qu'ils aient été objets de science ou de curiosité, les restes humains patrimonialisés bénéficient depuis quelques dizaines d'années d'un regain d'intérêt certain qui se caractérise par une revalorisation croissante de leur potentiel scientifique et historique au travers de projets de recherche ou d'expositions temporaires réguliers ${ }^{1}$. À défaut d'un réel statut de sujet au sens juridique du terme ${ }^{2}$, c'est bien désormais la considération de leur condition d'individu longtemps laissée de côté qui tend à faire évoluer progressivement les pratiques scientifiques et conservatoires à leur endroit. Avertissements de plus en plus fréquents sur la présence de dépouilles humaines dans les expositions, cérémonies de restitution aux communautés d'origine, campagnes de reconditionnement peuvent être vus chacun à leur niveau comme autant de marques de respect du corps et de la dignité de l'individu dans le cadre des activités muséales ${ }^{3}$.

Cette évolution des pratiques, reflet d'un changement de sensibilité et de valeur, amène ainsi à considérer sous un jour nouveau et à prendre en considération des questionnements que l'on n'avait pas auparavant. De manière plus évidente et plus directe que pour d'autres types de collection, l'évolution de la considération à l'endroit de ces «spécimens » redevenus « individus » reflète en effet celle de la société par rapport au corps, à la mort, à l'Autre, et ne peut être dissociée de débats et discussions plus larges qui touchent à notre passé colonial, au consentement et à la donation du corps, ainsi qu'à toutes les questions philosophiques ou religieuses ayant trait à ces thèmes universels.

Ce changement de regard n'épargne pas le domaine de la conservation-restauration et invite à un certain examen de conscience par rapport aux pratiques passées dans leur ensemble. Les restes humains ne peuvent décemment plus être considérés comme des objets et, à ce titre, questionnent les fondements mêmes de notre discipline ou tout du moins ses limites, conférant au travail sur ces collections une dimension éthique et morale prégnante par rapport à d'autres biens culturels.

\section{Multiplicité des usages et orientations des besoins}

Têtes ou membres désolidarisés, enfoncements au niveau des zones de préhension, fentes de dessiccations, décoloration, attaques d'insectes : la fragilité intrinsèque des restes humains combinée à des pratiques muséales ou autres peu précautionneuses sont à l'origine d'une multitude de dégradations 


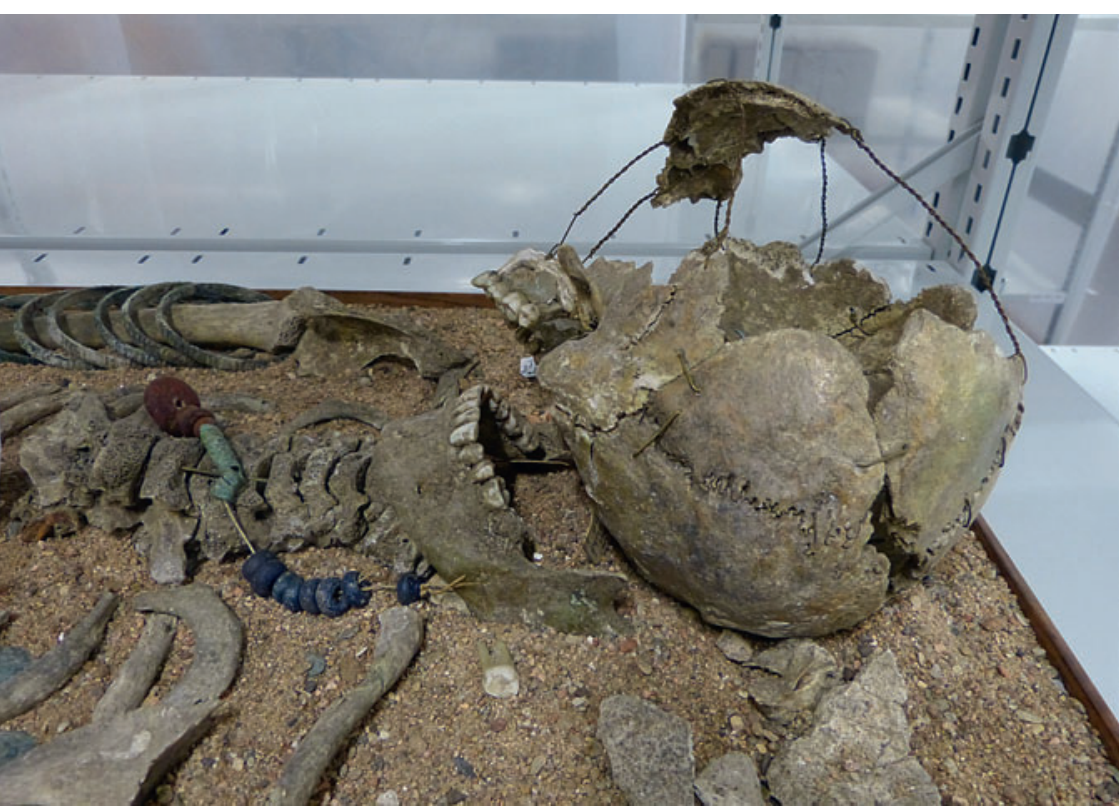

Fig. 2. Évocation épurée au point d'en perdre la lisibilité. Sépulture de Peyre-Haute, musée des Confluences. (c) L. Cadot.

palliées avec plus ou moins de succès. Si les interventions de « remise en état », suite à des avaries liées à ce que l'on qualifie aujourd'hui de «mauvaises pratiques » ou de négligence, se retrouvent régulièrement dans les collections, toutes les interventions passées ne relèvent pourtant pas de la restauration à proprement parler, mais constituent des réponses issues des paradigmes de leur époque aux besoins de l'exposition, de l'enseignement ou de l'étude.

À défaut de rapports d'intervention ou a minima de mentions écrites dans des inventaires ou des publications, les traces de ces interventions constituent en elles-mêmes la source première pour prendre la mesure de ces pratiques. Les considérations présentées dans ces lignes résultent, de fait, de nos propres observations et réflexions accumulées au fil de visites et de projets d'étude ou de conservation au cours des dix dernières années. Loin de fournir un panorama général et exhaustif, nous proposons ici quelques cas ponctuels, mais néanmoins suffisamment récurrents et significatifs pour voir se dessiner un ensemble de pratiques qui nous ont interpelées et interrogées.

Les contours de cette « brève histoire de la conservation-restauration des restes humains » semblent de prime abord varier d'un type de collection à un autre, marquant la pluralité des pratiques en fréquence et motivation selon les usages institutionnels. Sans surprise, les dépouilles à valeur esthétique et marchande-momies égyptiennes et têtes trophées en premier lieu - ayant souvent transité par les cabinets d'amateurs ou le marché de l'art, présentent fréquemment des traces d'interventions cherchant à maintenir leur aspect ou tout du moins leur cohésion (fig. 5 a). Qu'il s'agisse de consolidations, de comblements, de refixage, de soclages ou autre, tous ces traitements visent en général à les mettre en valeur dans une optique de présentation.
Les collections archéologiques et anthropologiques faisant l'objet de consultations ou d'expositions régulières peuvent présenter pour leur part des remontages ou restitutions plus ou moins invasives, afin de faciliter les manipulations et prises de mesure et d'offrir au public des crânes complets ou des squelettes remis en continuité anatomique plus évocateurs (fig. 1). Ceci semble particulièrement vrai concernant les ossements fossiles souvent fragmentaires et lacunaires au moment de leur découverte. Des reconstitutions d'ensembles funéraires comme la tombe de Téviec du Muséum de Toulouse participent également de ce processus de diffusion, au point que les ajouts muséographiques associés aux restes archéologiques dans les années 1930 sont aujourd'hui conservés au même titre que les ossements eux-mêmes (fig. 2). La présence de dispositifs de suspension ou de manipulation tels que socles (fig. 3) ou potences rappelle la vocation première de ces collections pensées au moment de leur constitution comme des supports d'enseignement et de démonstration. Dans ces différents cas de figure, les pratiques conservatoires sont là pour faciliter les usages muséaux autant que pour réparer les dégâts causés par ces mêmes usages.

Dans ce bref aperçu des interventions à valeur principalement esthétique ou scientifique, les collections médicales font figure d'exception par le caractère très limité, voire l'absence de traitement de restauration à proprement parler sur les préparations conservées. Cet état de fait s'explique sans doute autant par le caractère renouvelable de ces collections produites en majorité par et pour l'enseignement ${ }^{4}$ que par l'éloignement de la sphère muséale des médecins souvent en charge de ces collections.

Il est à l'heure actuelle très difficile d'établir une chronologie ou d'attribuer avec certitude ces interventions à un type d'intervenant plutôt qu'à un autre faute de documentation et de recherche sur la question ${ }^{5}$. Toutefois, l'existence 


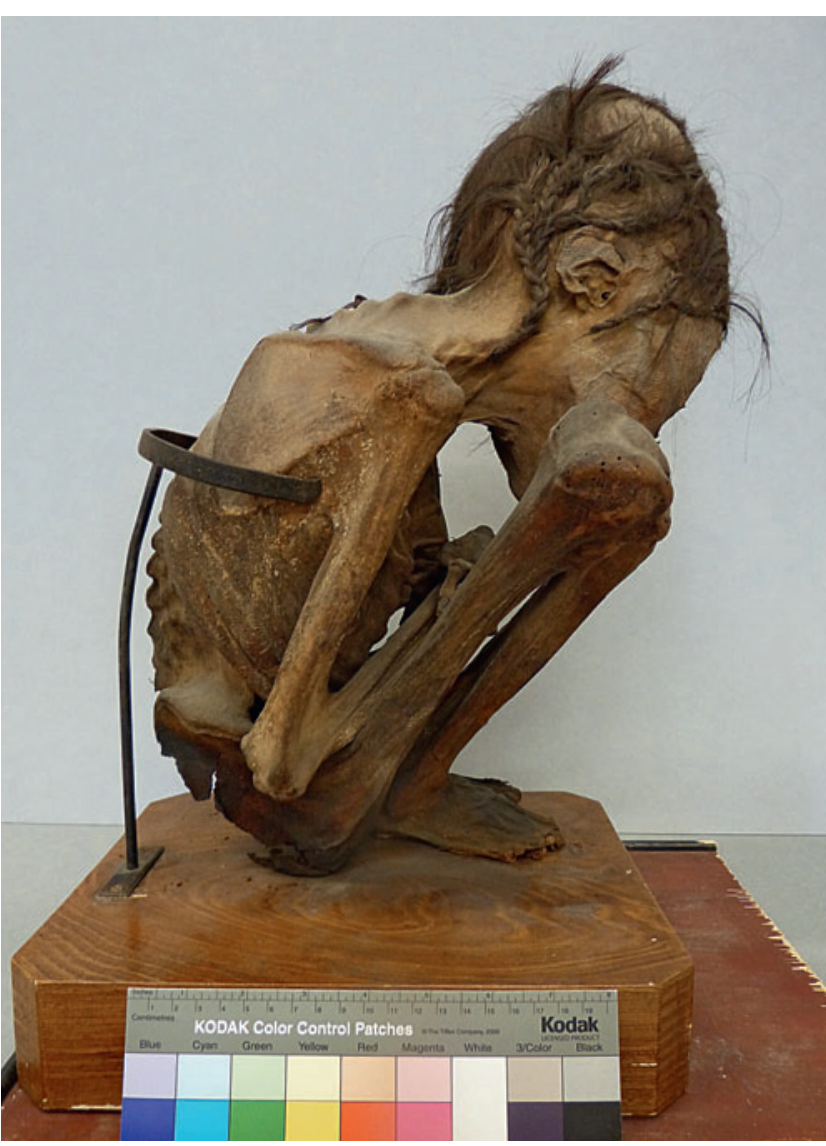

Fig. 3. Momie d'enfant soclée, Muséum d'histoire naturelle de Toulouse. (C) L. Cadot.

relativement récente de professionnels formés susceptibles d'intervenir sur ces collections laisse supposer que les archéologues aussi bien que les chercheurs, conservateurs, préparateurs, bénévoles, ou même, hors institution, les marchands et les collectionneurs puissent être indifféremment à l'origine des traitements observés.

\section{Une pratique empirique de la conservation sur fond de paradigmes changeants}

S'il est impossible de se mettre à la place de nos prédécesseurs pour comprendre leur démarche, il semble en revanche assez évident que les restaurations passées relèvent davantage de la réparation, voire de la restitution selon les acceptions proposées par Jacques Cuisin pour les naturalia ${ }^{6}$, que de la restauration telle qu'on peut l'envisager aujourd'hui. On observe en effet bien souvent une forme de maladresse dans la mise en ouvre de ces interventions, soit qu'elles aient été réalisées avec les moyens du bord, soit que des techniques empruntées à la restauration des œuvres d'art viennent donner l'impression d'une inadéquation entre la réalisation et le but recherché (fig. 4). L'hétérogénéité des matériaux de restauration - colles, vernis, charges de comblements ou autres - et la disparité des techniques

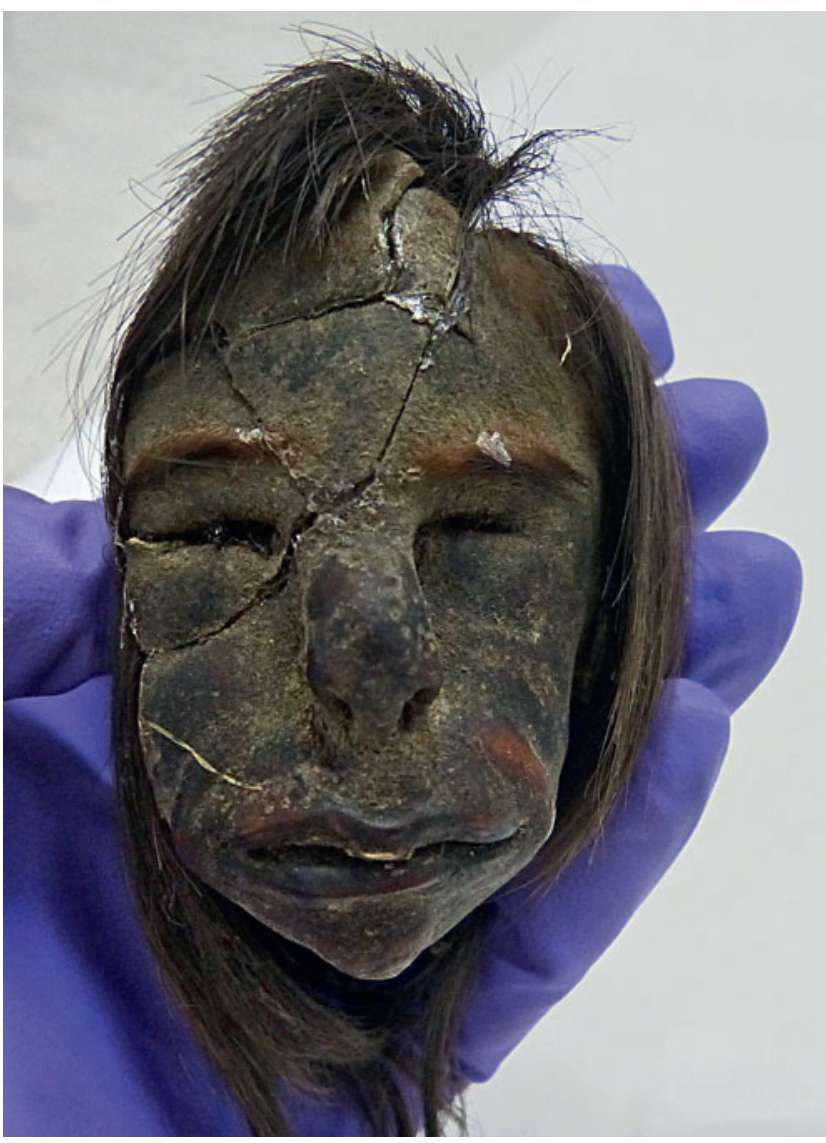

Fig. 4. Tête réduite brisée et recollée, musée du quai Branly, ancienne collection du laboratoire d'ethnologie du musée de l'Homme. (C) L. Cadot.

renforcent cette impression d'empirisme. Si ces interventions, comme les traitements insecticides qui semblent avoir largement contaminé les collections, nous posent aujourd'hui problème par leur manque de réversibilité ou d'innocuité, il ne faut pas oublier que ce souci d'assurer leur pérennité a malgré tout permis de préserver nombre de dépouilles.

Au demeurant, les altérations les plus spectaculaires observées sur les restes humains patrimonialisés ne découlent pas tant de déficiences conservatoires - encore qu'une certaine forme d'abandon de spécimens soit à l'origine de dégâts aussi spectaculaires que désolants - que de la prépondérance de la recherche sur ces collections pendant tout le XIX ${ }^{\mathrm{e}}$ et une bonne partie du $\mathrm{XX}^{\mathrm{e}}$ siècle. L'observation des traces anciennes de démaillotage ou de déshabillage de momies, de dissection ou de démembrement traduit bien la « chosification » des restes et l'importance toute secondaire portée au maintien de l'intégrité au profit des besoins liés à l'investigation. Cette primauté de la recherche inclut certaines pratiques jugées peu précautionneuses aujourd'hui comme les prélèvements d'os, de peau ou de cheveux, qui nous semblent, à la lumière des possibilités technologiques actuelles, bien disproportionnées.

Les interprétations dans le remontage des squelettes et plus particulièrement des crânes - partie du corps ô combien mise en avant - posent quant à elles la question de la 

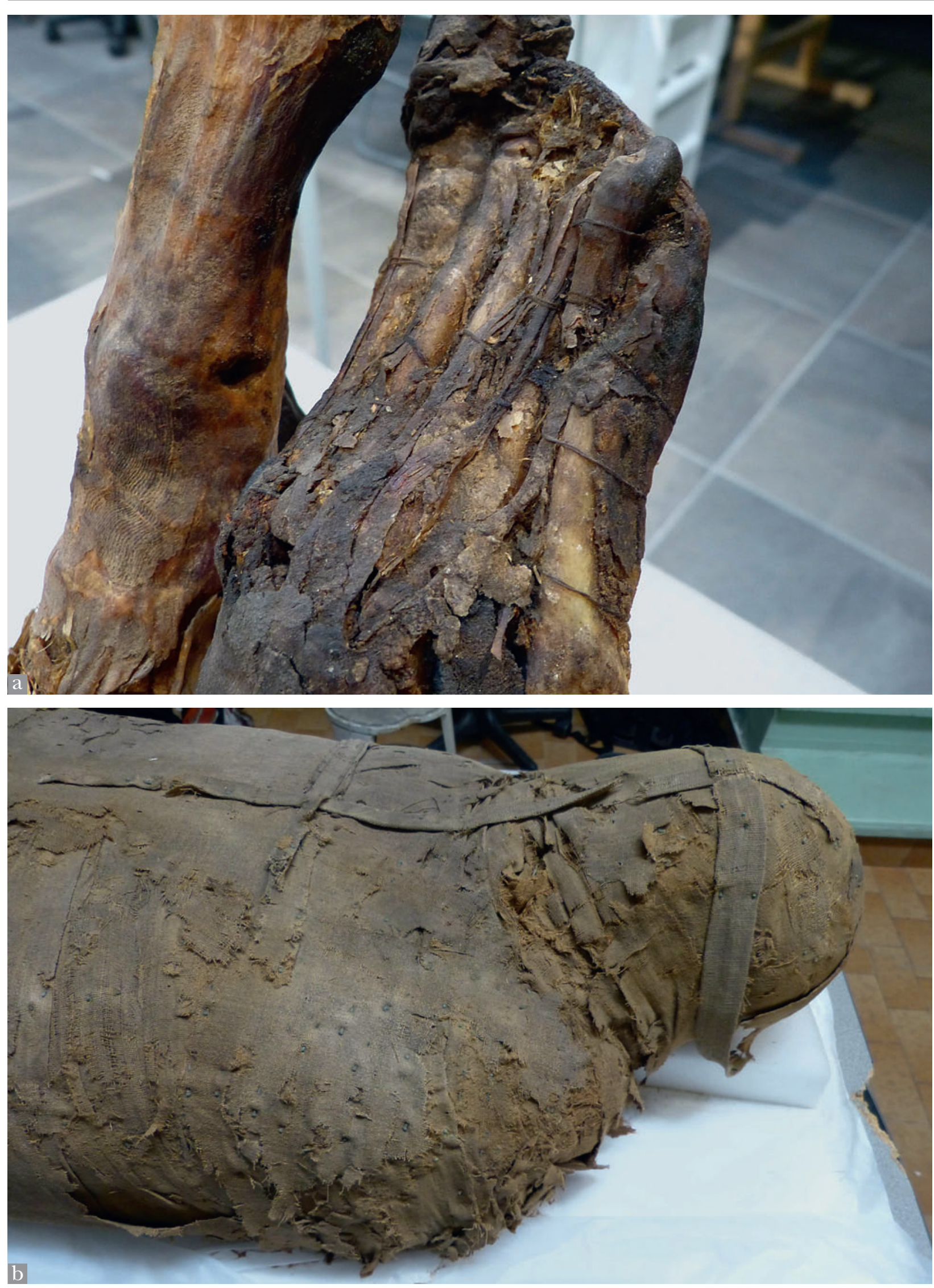

Fig. 5. a : Exemple de réparation pratiquée vraisemblablement au moment de la fouille sur un orteil de momie, Fondazione Museo delle Antichità Egizie, Turin. b : Bandelettes maintenues par des épingles modernes aujourd'hui corrodées, musée Déchelette, Roanne. @ L. Cadot. 
subjectivité des fondements scientifiques de ces pratiques. À titre d'exemple, le crâne de l'homme de la Chapelle-aux-Saints dont la version remontée par Marcellin Bouleau au début des années 1910 fut guidée par un certain nombre d'a priori, a en effet contribué à véhiculer une vision sauvage et fruste des Néanderthaliens, aujourd'hui corrigée par la découverte de nouveaux fossiles?.

De tout ceci ressort un sentiment contrasté d'inégalité de considération et de moyens mis en œuvre au cours du temps pour permettre la transmission de ces restes, entre appropriation des collections par ceux qui les ont constitué et/ou étudié et périodes de délaissement.

\section{Comprendre les pratiques passées pour guider les pratiques actuelles}

En matière de restauration, porter un regard rétrospectif sur les pratiques antérieures participe de la construction même de la discipline en grande partie fondée sur le retour d'expérience et l'évaluation des traitements dans la durée. Concernant les restes humains, ce bel esprit critique peut facilement glisser vers le jugement de valeur et il est parfois difficile de s'en départir pour comprendre les motivations et la démarche mise en œuvre par nos prédécesseurs. Quand on pense «anciennes restaurations », c'est le mot «bricolage » qui sonne en écho comme une manière de mettre à distance des pratiques qui ne sont pas estampillées du sceau de notre déontologie actuelle. Car ce sont bien les principes et valeurs de la discipline fixés dans les codes professionnels qui nous permettent aujourd'hui de justifier nos propres interventions sur ces sujets patrimoniaux dont la détention par les musées est loin de faire l'unanimité et se trouve parfois remise en cause.

Face à ces pratiques disparates, on aura tendance à considérer que « le mal est fait ». Il faut bien reconnaître que leur réversibilité est souvent très limitée, voire inexistante. De même, les degrés plus ou moins importants de dégradation ou de contamination par l'apport d'éléments exogènes potentiellement perturbateurs (fig. 5 b), participent de la confusion ou de la détérioration, pouvant aller jusqu'à la disparition pure et simple d'informations macro- ou microscopiques. Tous ces aspects incitent à l'intégration d'un principe de précaution fondé sur le minimalisme strict des interventions dans l'optique de préserver le potentiel d'études, d'analyses ou d'examens futurs.

Indépendamment des difficultés techniques qui peuvent se présenter, la question de la dérestauration se trouve par ailleurs grandement limitée du point de vue éthique en ce qu'elle constitue un niveau d'intervention supplémentaire dans l'histoire du traitement de ces restes, autant que par l'aspect désormais documentaire de ces pratiques anciennes du point de vue de l'histoire des collections. Elle ne se justifiera dès lors que par son aspect curatif visant à stabiliser un processus évolutif de dégradation causé par une intervention antérieure, chaque prise de décision mettant en avant le particularisme ontologique des restes humains, mais aussi le rapport sensible du praticien et des responsables scientifiques à ces collections à part.
Notes

1. Citons l'exposition Quatre momies et demie en 2015 au musée Déchelette de Roanne, ou bien encore la synthèse des réflexions et pratiques mises en place au musée des Confluences, Les cahiers du musée des Confluences (collectif, 2012).

2. Les restes humains demeurent des choses en termes de qualification juridique. Cornu, 2010, p. 44-45.

3. À titre d'exemple, les récentes rénovations des parcours permanents du musée des Confluences et du musée de l'Homme ont ainsi constitué, pour ces deux grandes institutions, l'occasion de sonder le contenu de leurs collections et de réfléchir à la manière la plus opportune de présenter ces dépouilles tantôt fascinantes, tantôt dérangeantes - voire embarrassantes -, mais toujours plébiscitées par le public.

4. On n'hésitait pas en effet à remplacer les préparations et spécimens obsolètes ou en mauvais état.

5. Parmi les trop rares recherches sur le sujet, citons le travail de Pauline Carminati (Carminati, 2011) sur la collection des momies du musée de l'Homme.
6. Cuisin, 2005, p. 14.

7. Nous remercions Mathilde Vauquelin, élève à l'École du Louvre, pour son étude de cas sur ce crâne dont un nouveau remontage a été réalisé dans les années 1980 .

\section{Bibliographie}

Cadot L., 2015, « Actualité récente et évolution des pratiques autour des restes humains patrimonialisés ", La Lettre de l'OCIM, n ${ }^{\circ} 157$, p. 32-34.

Cadot L., 2012, « La conservationrestauration des restes humains en question ", Coré, n 27, p. 3-11.

Carminati P., 2011, « Les momies du Muséum national d'histoire naturelle : du cabinet anthropologique au musée de l'Homme ", La Lettre de l'OCIM, $\mathrm{n}^{\circ} 137$, p. 26-34.

Cassman V., Odegaard N., Powell J., 2006, Human remains: Guide for Museums and Academic Institutions, Altamira Press, Lanham.
Cornu M., 2010, « La condition juridique des restes humains, l'évolution récente du contexte légal ", Musées et Collections publiques de France, $\mathrm{n}^{\circ} 259$.

Cuisin J., 2005, « Être ou ne plus être, quelle restauration pour les naturalia?", $C R B C, \mathrm{n}^{\circ} 23$, p. 3-15.

Odegaard N., Sadongei A., 2005, Old poisons, new problems, managing contaminated cultural materials, Altamira press, Lanham.

Vuissoz A., «L'effet des traitements de conservation restauration sur l'acide désoxyribonucléique de cuir archéologique ", CRBC, n²3, p. 67- 69

Collectif, 2012, «Les restes humains au Musée des Confluences ", Les cahiers du Musée des Confluences, études scientifiques $\mathrm{n}^{\circ} 3$, p. 7-64.

Panagiaris G., 2001, "The Influence of Conservation Treatments on Physical Anthropology Research”, Human Remains, Conservation, Retrieval and Analysis, Proceedings of a Conference held in Williamsburg, Nov 7-11th 1999, BAR International Series 934, p. 95-98. 